\title{
New Examples of Oriented Matroids with Disconnected Realization Spaces
}

\author{
Yasuyuki Tsukamoto
}

Received: 3 February 2012 / Revised: 15 July 2012 / Accepted: 31 August 2012 /

Published online: 12 October 2012

(C) Springer Science+Business Media, LLC 2012

\begin{abstract}
We construct oriented matroids of rank 3 on 13 points whose realization spaces are disconnected. They are defined on smaller point-sets than the known examples with this property. Moreover, we construct one on 13 points whose realization space is a connected but non-irreducible semialgebraic variety.
\end{abstract}

Keywords Oriented matroids $\cdot$ Realization space

\section{Oriented Matroids and Matrices}

Throughout this section, we fix positive integers $r$ and $n$.

Let $X=\left(x_{1}, \ldots, x_{n}\right) \in \mathbb{R}^{r n}$ be a real $(r, n)$ matrix of rank $r$, and $E=\{1, \ldots, n\}$ be the set of labels of the columns of $X$. For such a matrix $X$, a map $\mathcal{X}_{X}$ can be defined as

$$
\mathcal{X}_{X}: E^{r} \rightarrow\{-1,0,+1\}, \quad \mathcal{X}_{X}\left(i_{1}, \ldots, i_{r}\right):=\operatorname{sgn} \operatorname{det}\left(x_{i_{1}}, \ldots, x_{i_{r}}\right) .
$$

The map $\mathcal{X}_{X}$ is called the chirotope of $X$. The chirotope $\mathcal{X}_{X}$ encodes the information regarding the combinatorial type, which is called the oriented matroid of $X$. In this case, the oriented matroid determined by $\mathcal{X}_{X}$ is of rank $r$ on $E$.

We note some properties which the chirotope $\mathcal{X}_{X}$ of a matrix $X$ satisfies.

1. $\mathcal{X}_{X}$ is not identically zero.

2. $\mathcal{X}_{X}$ is alternating, i.e. $\mathcal{X}_{X}\left(i_{\sigma(1)}, \ldots, i_{\sigma(r)}\right)=\operatorname{sgn}(\sigma) \mathcal{X}_{X}\left(i_{1}, \ldots, i_{r}\right)$ for all $i_{1}, \ldots$, $i_{r} \in E$ and all permutations $\sigma$.

3. For all $i_{1}, \ldots, i_{r}, j_{1}, \ldots, j_{r} \in E$ such that $\mathcal{X}_{X}\left(j_{k}, i_{2}, \ldots, i_{r}\right) \cdot \mathcal{X}_{X}\left(j_{1}, \ldots, j_{k-1}, i_{1}\right.$, $\left.j_{k+1}, \ldots, j_{r}\right) \geq 0$ for $k=1, \ldots, r$, we have $\mathcal{X}_{X}\left(i_{1}, \ldots, i_{r}\right) \cdot \mathcal{X}_{X}\left(j_{1}, \ldots, j_{r}\right) \geq 0$.

Y. Tsukamoto $(\bowtie)$

Department of Human Coexistence, Kyoto University, Sakyo-ku, Kyoto 606-8501, Japan

e-mail: tsukamoto@i.h.kyoto-u.ac.jp 
The third property follows from the identity

$$
\begin{aligned}
& \operatorname{det}\left(x_{1}, \ldots, x_{r}\right) \cdot \operatorname{det}\left(y_{1}, \ldots, y_{r}\right) \\
& \quad=\sum_{k=1}^{r} \operatorname{det}\left(y_{k}, x_{2}, \ldots, x_{r}\right) \cdot \operatorname{det}\left(y_{1}, \ldots, y_{k-1}, x_{1}, y_{k+1}, \ldots, y_{r}\right), \\
& \quad \text { for all } x_{1}, \ldots, x_{r}, y_{1}, \ldots, y_{r} \in \mathbb{R}^{r} .
\end{aligned}
$$

Generally, an oriented matroid of rank $r$ on $E$ ( $n$ points) is defined by a map $\chi: E^{r} \rightarrow\{-1,0,+1\}$, which satisfies the above three properties ([1]). The map $\chi$ is also called the chirotope of an oriented matroid. We use the notation $\mathcal{M}(E, \chi)$ for an oriented matroid which is on the set $E$ and is defined by the chirotope $\chi$.

An oriented matroid $\mathcal{M}(E, \chi)$ is called realizable or constructible, if there exists a matrix $X$ such that $\chi=\mathcal{X}_{X}$. Not all oriented matroids are realizable, but we do not consider the non-realizable case in this paper.

Definition 1.1 A realization of an oriented matroid $\mathcal{M}=\mathcal{M}(E, \chi)$ is a matrix $X$ such that $\mathcal{X}_{X}=\chi$ or $\mathcal{X}_{X}=-\chi$.

Two realizations $X, X^{\prime}$ of $\mathcal{M}$ are called linearly equivalent, if there exists a linear transformation $A \in G L(r, \mathbb{R})$ such that $X^{\prime}=A X$. Here we have the equation $\mathcal{X}_{X^{\prime}}=$ $\operatorname{sgn}(\operatorname{det} A) \cdot \mathcal{X}_{X}$.

Definition 1.2 The realization space $\mathcal{R}(\mathcal{M})$ of an oriented matroid $\mathcal{M}$ is the set of all linearly equivalent classes of realizations of $\mathcal{M}$, in the quotient topology induced from $\mathbb{R}^{r n}$.

Our motivation is as follows: In 1956, Ringel asked whether the realization spaces $\mathcal{R}(\mathcal{M})$ are necessarily connected [6]. It is known that every oriented matroid on less than nine points has a contractible realization space. In 1988, Mnëv showed that $\mathcal{R}(\mathcal{M})$ can be homotopy equivalent to an arbitrary semialgebraic variety [3]. His result implies that they can have arbitrary complicated topological types. In particular, there exist oriented matroids with disconnected realization spaces. Suvorov and Richter-Gebert constructed such examples of oriented matroids of rank 3 on 14 points, in 1988 and in 1996, respectively [5, 7]. However, it is unknown which is the smallest number of points on which oriented matroids can have disconnected realization spaces. See [1] for more historical comments.

One of the main results of this paper is the following.

Theorem 1.3 There exist oriented matroids of rank 3 on 13 points whose realization spaces are disconnected.

Let $d$ and $p$ be positive integers. The solution of a finite number of polynomial equations and polynomial strict inequalities with integer coefficients on $\mathbb{R}^{d}$ is called an elementary semialgebraic set.

Let $f_{1}, \ldots, f_{p} \in \mathbb{Z}\left[v_{1}, \ldots, v_{d}\right]$ be polynomial functions on $\mathbb{R}^{d}$, and $V \subset \mathbb{R}^{d}$ be an elementary semialgebraic set. For a $p$-tuple $\varepsilon=\left(\varepsilon_{1}, \ldots, \varepsilon_{p}\right) \in\{-, 0,+\}^{p}$, let

$$
V_{\varepsilon}:=\left\{v \in V \mid \operatorname{sgn}\left(f_{i}(v)\right)=\varepsilon_{i} \text { for } i=1, \ldots, p\right\}
$$


denote the corresponding subset of $V$. The collection of the elementary semialgebraic sets $\left(V_{\varepsilon}\right)_{\varepsilon \in\{-, 0,+\}^{p}}$ is called a partition of $V$.

In the case $r=3$, a triple $(i, j, k) \in E^{3}$ is called a basis of $\chi$ if $\chi(i, j, k) \neq 0$. Let $B=(i, j, k)$ be a basis of $\chi$ such that $\chi(B)=+1$. The realization space of an oriented matroid $\mathcal{M}=\mathcal{M}(E, \chi)$ of rank 3 can be given by an elementary semialgebraic set

$$
\mathcal{R}(\mathcal{M}, B):=\left\{X \in \mathbb{R}^{3 n} \mid x_{i}=e_{1}, x_{j}=e_{2}, x_{k}=e_{3}, \mathcal{X}_{X}=\chi\right\},
$$

where $e_{1}, e_{2}, e_{3}$ are the fundamental vectors of $\mathbb{R}^{3}$. For another choice of basis $B^{\prime}$ of $\chi$, we have a rational isomorphism between $\mathcal{R}(\mathcal{M}, B)$ and $\mathcal{R}\left(\mathcal{M}, B^{\prime}\right)$. Therefore, realization spaces of oriented matroids are semialgebraic varieties.

The universal partition theorem states that, for every partition $\left(V_{\varepsilon}\right)_{\varepsilon \in\{-, 0,+\}^{p}}$ of $\mathbb{R}^{d}$, there exists a family of oriented matroids $\left(\mathcal{M}^{\varepsilon}\right)_{\varepsilon \in\{-, 0,+\}^{p}}$ such that the collection of their realization spaces with a common basis $\left(\mathcal{R}\left(\mathcal{M}^{\varepsilon}, B\right)\right)_{\varepsilon \in\{-, 0,+\}}$ is stably

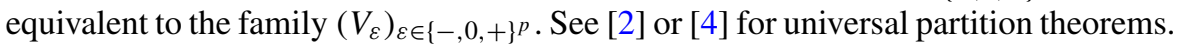

We construct three oriented matroids $\mathcal{M}^{\varepsilon}$ with $\varepsilon \in\{-, 0,+\}$ of rank 3 on 13 points, whose chirotopes differ by a sign on a certain triple. These oriented matroids present a partial oriented matroid with the sign of a single base non-fixed, whose realization space is partitioned by fixing the sign of this base. The two spaces $\mathcal{R}\left(\mathcal{M}^{-}\right)$ and $\mathcal{R}\left(\mathcal{M}^{+}\right)$are disconnected, and $\mathcal{R}\left(\mathcal{M}^{0}\right)$, which is a wall between the two, is connected but non-irreducible. So we also have the following.

Theorem 1.4 There exists an oriented matroid of rank 3 on 13 points whose realization space is connected but non-irreducible.

Remark 1.5 An oriented matroid $\mathcal{M}(E, \chi)$ is called uniform if it satisfies $\chi\left(i_{1}, \ldots\right.$, $\left.i_{r}\right) \neq 0$ for all $i_{1}<\cdots<i_{r} \in E$. Suvorov's example on 14 points is uniform, and the examples which we construct are non-uniform. It is still unknown whether there exists a uniform oriented matroid on less than 14 points with a disconnected realization space.

\section{Construction of the Examples}

Throughout this section, we set $E=\{1, \ldots, 13\}$.

Let $X(s, t, u)$ be a real $(3,13)$ matrix with three parameters $s, t, u \in \mathbb{R}$ given by

$$
\begin{aligned}
& X(s, t, u) \\
& :=\left(x_{1}, \ldots, x_{13}\right) \\
& =\left(\begin{array}{lllllllll}
1 & 0 & 0 & 1 & s & s & 0 & 1 & 1 \\
0 & 1 & 0 & 1 & 0 & 1 & t & t & u \\
0 & 0 & 1 & 1 & 1 & 1 & 1 & 1 & 0
\end{array}\right. \\
& \left.\begin{array}{cccc}
s t & s+t-u-s t+s u & s+t-s t-s^{2} u & s(t-u+s u) \\
t & t-u+s u & t & t-u+s u \\
1-s u & 1-u+s u & 1-s u & 1-u+s u
\end{array}\right) \text {. }
\end{aligned}
$$


This is a consequence of the computation of the following construction sequence. Both operations " $\vee$ " and " $\wedge$ " can be computed in terms of the standard cross product " $x$ " in $\mathbb{R}^{3}$. The whole construction depends only on the choice of the three parameters $s, t, u \in \mathbb{R}$. We have

$$
\begin{aligned}
x_{1} & ={ }^{t}(1,0,0), \quad x_{2}={ }^{t}(0,1,0), \\
x_{3} & ={ }^{t}(0,0,1), \quad x_{4}={ }^{t}(1,1,1), \\
x_{5} & =s \cdot x_{1}+x_{3}, \\
x_{6} & =\left(x_{1} \vee x_{4}\right) \wedge\left(x_{2} \vee x_{5}\right), \\
x_{7} & =t \cdot x_{2}+x_{3}, \\
x_{8} & =\left(x_{1} \vee x_{7}\right) \wedge\left(x_{2} \vee x_{4}\right), \\
x_{9} & =u \cdot x_{2}+x_{1}, \\
x_{10} & =\left(x_{7} \vee x_{9}\right) \wedge\left(x_{3} \vee x_{6}\right), \\
x_{11} & =\left(x_{4} \vee x_{5}\right) \wedge\left(x_{8} \vee x_{9}\right), \\
x_{12} & =\left(x_{1} \vee x_{10}\right) \wedge\left(x_{4} \vee x_{5}\right), \\
x_{13} & =\left(x_{3} \vee x_{6}\right) \wedge\left(x_{1} \vee x_{11}\right) .
\end{aligned}
$$

We set $X_{0}=X\left(\frac{1}{2}, \frac{1}{2}, \frac{1}{3}\right)$. The chirotope $\chi^{\varepsilon}$ is the alternating map such that

$$
\begin{gathered}
\chi^{\varepsilon}(i, j, k)= \begin{cases}\varepsilon & \text { if }(i, j, k)=(9,12,13), \\
\mathcal{X}_{X_{0}}(i, j, k) & \text { otherwise, }\end{cases} \\
\text { for all }(i, j, k) \in E^{3}(i<j<k),
\end{gathered}
$$

where $\varepsilon \in\{-, 0,+\}$.

The oriented matroid which we will study is $\mathcal{M}^{\varepsilon}:=\mathcal{M}\left(E, \chi^{\varepsilon}\right)$.

Remark 2.1 We can replace $X_{0}$ with $X\left(\frac{1}{2}, \frac{1}{2}, u^{\prime}\right)$ where $u^{\prime}$ is chosen from $\mathbb{R} \backslash\{-1,0$, $\left.\frac{1}{2}, 1, \frac{3}{2}, 2,3\right\}$. We will study the case $0<u^{\prime}<\frac{1}{2}$. If we choose $u^{\prime}$ otherwise, we can get other oriented matroids with disconnected realization spaces.

In the construction sequence, we need no assumption on the collinearity of $x_{9}, x_{12}, x_{13}$. Hence every realization of $\mathcal{M}^{\varepsilon}$ is linearly equivalent to a matrix $X(s, t, u)$ for certain $s, t, u$, up to multiplication on each column with positive scalar.

Moreover, we have the rational isomorphism

$$
\mathcal{R}^{*}\left(\chi^{\varepsilon}\right) \times(0, \infty)^{12} \cong \mathcal{R}\left(\mathcal{M}^{\varepsilon}\right),
$$

where $\mathcal{R}^{*}\left(\chi^{\varepsilon}\right):=\left\{(s, t, u) \in \mathbb{R}^{3} \mid \mathcal{X}_{X(s, t, u)}=\chi^{\varepsilon}\right\}$. Thus we have only to prove that the set $\mathcal{R}^{*}\left(\chi^{\varepsilon}\right)$ is disconnected (resp. non-irreducible) to show that the realization space $\mathcal{R}\left(\mathcal{M}^{\varepsilon}\right)$ is disconnected (resp. non-irreducible).

The equation $\mathcal{X}_{X(s, t, u)}=\chi^{\varepsilon}$ means that

$$
\operatorname{sgn} \operatorname{det}\left(x_{i}, x_{j}, x_{k}\right)=\chi^{\varepsilon}(i, j, k), \quad \text { for all }(i, j, k) \in E^{3} .
$$


Fig. 1 Column vectors of $X_{0}$

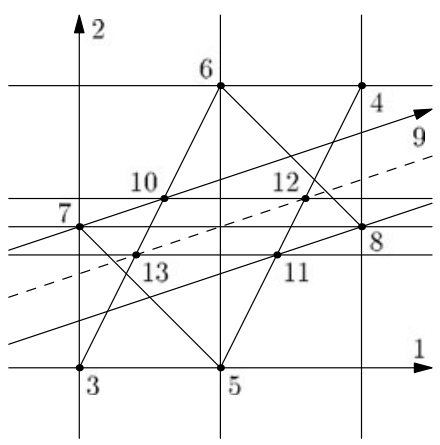

We write some of them which give the equations on the parameters $s, t, u$. Note that for all $(i, j, k) \in E^{3}(\{i, j, k\} \neq\{9,12,13\})$, the sign is given by

$$
\chi^{\varepsilon}(i, j, k)=\left.\operatorname{sgn} \operatorname{det}\left(x_{i}, x_{j}, x_{k}\right)\right|_{s=t=1 / 2, u=1 / 3} .
$$

From the equation $\operatorname{sgn} \operatorname{det}\left(x_{2}, x_{3}, x_{5}\right)=\operatorname{sgn}(s)=\operatorname{sgn}(1 / 2)=+1$, we get $s>0$. Similarly, we get $\operatorname{det}\left(x_{2}, x_{5}, x_{4}\right)=1-s>0$; therefore,

$$
0<s<1 \text {. }
$$

From the equations $\operatorname{det}\left(x_{1}, x_{7}, x_{3}\right)=t>0, \operatorname{det}\left(x_{1}, x_{4}, x_{7}\right)=1-t>0$, we get

$$
0<t<1 \text {. }
$$

Moreover, we have the inequalities

$$
\begin{aligned}
\operatorname{det}\left(x_{1}, x_{9}, x_{3}\right) & =u>0, \\
\operatorname{det}\left(x_{4}, x_{7}, x_{9}\right) & =1-t-u>0, \\
\operatorname{det}\left(x_{3}, x_{9}, x_{8}\right) & =t-u>0, \\
\operatorname{det}\left(x_{5}, x_{13}, x_{7}\right) & =s\left(t^{2}-(1-s) u\right)>0, \\
\operatorname{det}\left(x_{6}, x_{12}, x_{8}\right) & =(1-s)\left((1-t)^{2}-s u\right)>0 .
\end{aligned}
$$

From the equation $\operatorname{det}\left(x_{9}, x_{12}, x_{13}\right)=u(1-2 s)(1-2 t+t u-s u)$, we get

$$
\operatorname{sgn}(u(1-2 s)(1-2 t+t u-s u))=\varepsilon .
$$

Conversely, if we have Eqs. (2)-(9), then we get (1).

We can interpret a $(3,13)$ matrix as the set of vectors $\left\{x_{1}, \ldots, x_{13}\right\} \subset \mathbb{R}^{3}$. After we normalize the last coordinate for $x_{i}(i \in E \backslash\{1,2,9\})$, we can visualize the matrix on the affine plane $\left\{(x, y, 1) \in \mathbb{R}^{3}\right\} \cong \mathbb{R}^{2}$. Figure 1 shows the affine image of $X_{0}$. See Figs. 2, 3 for realizations of $\mathcal{M}^{\varepsilon}$.

Proof of Theorem 1.3 We prove that $\mathcal{R}^{*}\left(\chi^{-}\right)$and $\mathcal{R}^{*}\left(\chi^{+}\right)$are disconnected. From Eqs. (2)-(9), we obtain

$$
\mathcal{R}^{*}\left(\chi^{-}\right)=\left\{\begin{array}{l|c}
(s, t, u) \in \mathbb{R}^{3} \mid \begin{array}{c}
0<s<1,0<u<t<1-u, \\
(1-t)^{2}-s u>0, t^{2}-(1-s) u>0, \\
(1-2 s)(1-2 t+t u-s u)<0
\end{array}
\end{array}\right\},
$$



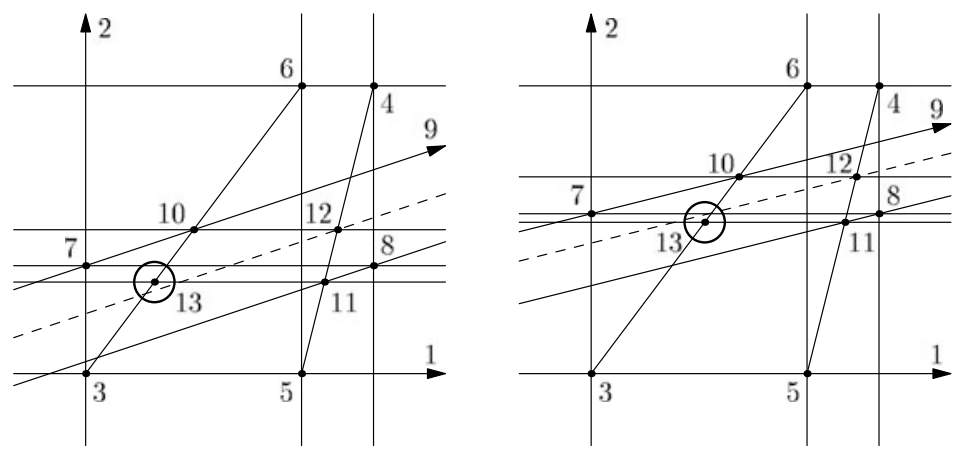

Fig. 2 Realization of $\mathcal{M}^{-}$(on the left) and that of $\mathcal{M}^{+}$(on the right)
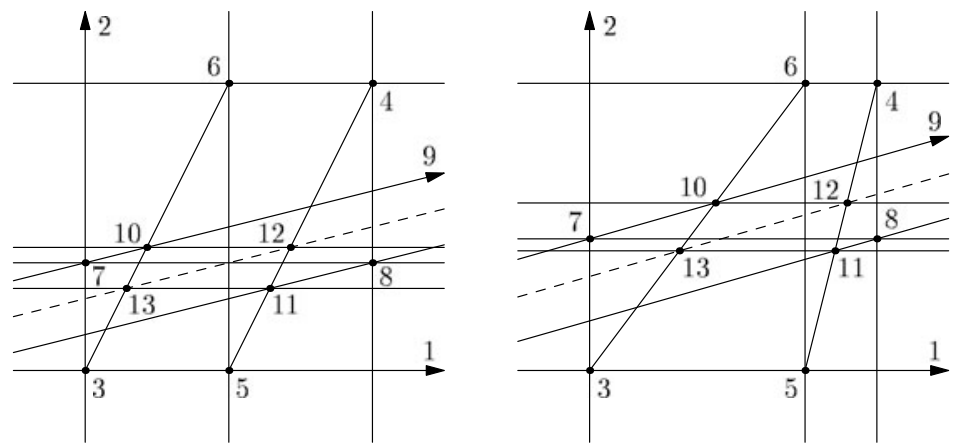

Fig. 3 Realizations of $\mathcal{M}^{0}$

$$
\mathcal{R}^{*}\left(\chi^{+}\right)=\left\{\begin{array}{l|c}
0<s<1,0<u<t<1-u \\
s, t, u) \in \mathbb{R}^{3} & \begin{array}{c}
(1-t)^{2}-s u>0, t^{2}-(1-s) u>0, \\
(1-2 s)(1-2 t+t u-s u)>0
\end{array}
\end{array}\right\} .
$$

First, we show that $\mathcal{R}^{*}\left(\chi^{-}\right)$is disconnected; more precisely, that it consists of two connected components. We do this by proving the next proposition.

\section{Proposition 2.2}

$$
\begin{aligned}
\mathcal{R}^{*}\left(\chi^{-}\right)= & \left\{(s, t, u) \in \mathbb{R}^{3} \mid \begin{array}{l}
0<s<1 / 2 \\
1 / 2<t<1
\end{array}, 0<u<\min \left\{1-t, \frac{(1-t)^{2}}{s}, \frac{2 t-1}{t-s}\right\}\right\} \\
& \cup\left\{(s, t, u) \in \mathbb{R}^{3} \mid \begin{array}{l}
1 / 2<s<1 \\
0<t<1 / 2
\end{array}, 0<u<\min \left\{t, \frac{t^{2}}{1-s}, \frac{1-2 t}{s-t}\right\}\right\} .
\end{aligned}
$$

Proof There are two cases:

$$
(1-2 s)(1-2 t+t u-s u)<0 \Leftrightarrow\left\{\begin{array}{l}
1-2 s>0,1-2 t+t u-s u<0, \\
\text { or } \\
1-2 s<0,1-2 t+t u-s u>0 .
\end{array}\right.
$$

Note that 


$$
\begin{aligned}
& (2-u)(2 t-1)=-2(1-2 t+t u-s u)+u(1-2 s) \\
& t^{2}-(1-s) u=-(1-2 t+t u-s u)+(1-t)(1-t-u), \\
& (1-t)^{2}-s u=(1-2 t+t u-s u)+t(t-u) .
\end{aligned}
$$

(C) For the case $1-2 s>0$ and $1-2 t+t u-s u<0$, the inequality $2 t-1>0$ follows from Eq. (10). Since we have $0<s<1 / 2<t<1$, we get

$$
\left\{\begin{array}{l}
1-2 t+t u-s u<0, \\
(1-t)^{2}-s u>0, \\
1-t-u>0
\end{array} \Leftrightarrow u<\min \left\{1-t, \frac{(1-t)^{2}}{s}, \frac{2 t-1}{t-s}\right\} .\right.
$$

For the other case $1-2 s<0$, similarly, we get $1-2 t>0$ from Eq. (10). Since we have $0<t<1 / 2<s<1$, we get

$$
\left\{\begin{array}{l}
1-2 t+t u-s u>0, \\
t^{2}-(1-s) u>0, \\
t-u>0
\end{array} \Leftrightarrow u<\min \left\{t, \frac{t^{2}}{1-s}, \frac{1-2 t}{s-t}\right\} .\right.
$$

(つ) For the component $0<s<1 / 2<t<1$, the inequalities $1-2 t+t u-s u<$ $0,(1-t)^{2}-s u>0,1-t-u>0$ follow from (13). Thus we get $t^{2}-(1-s) u>0$ from Eq. (11). The inequality $u<t$ holds because $t>1 / 2$ and $u<1-t$.

For the other component $0<t<1 / 2<s<1$, similarly, we get the inequalities $1-2 t+t u-s u>0, t^{2}-(1-s) u>0, t-u>0$ from (14), and $(1-t)^{2}-s u>0$ from Eq. (12). Last, we get $u<1-t$ from $t<1 / 2$ and $u<t$.

For the set $\mathcal{R}^{*}\left(\chi^{+}\right)$, we have the following proposition.

\section{Proposition 2.3}

$$
\begin{aligned}
\mathcal{R}^{*}\left(\chi^{+}\right)= & \left\{(s, t, u) \in \mathbb{R}^{3} \mid \begin{array}{c}
0<s<1 / 2,0<u<1 / 2, \\
(1-u)^{2}-(1-s) u>0,
\end{array} \sqrt{(1-s) u}<t<\frac{1-s u}{2-u}\right\} \\
& \cup\left\{(s, t, u) \in \mathbb{R}^{3} \mid \begin{array}{c}
1 / 2<s<1,0<u<1 / 2, \\
(1-u)^{2}-s u>0,
\end{array} \frac{1-s u}{2-u}<t<1-\sqrt{s u}\right\} .
\end{aligned}
$$

The proof is similar to that of Proposition 2.2 and is omitted.

Proof of Theorem 1.4 We show that $\mathcal{R}^{*}\left(\chi^{0}\right)$ consists of two irreducible components whose intersection is not empty. From Eqs. (2)-(9), we get

$$
\mathcal{R}^{*}\left(\chi^{0}\right)=\left\{\begin{array}{l|c}
0<s<1,0<u<t<1-u \\
(s, t, u) \in \mathbb{R}^{3} \mid \begin{array}{c}
2-t)^{2}-s u>0, t^{2}-(1-s) u>0 \\
(1-2 s)(1-2 t+t u-s u)=0
\end{array}
\end{array}\right\} .
$$

Here we have the decomposition

$$
\begin{aligned}
\mathcal{R}^{*}\left(\chi^{0}\right)= & \left\{(s, t, u) \in \mathbb{R}^{3} \mid 0<t<1,0<u<2 t^{2}, u<2(1-t)^{2}, 1-2 s=0\right\} \\
& \cup\left\{(s, t, u) \in \mathbb{R}^{3} \mid \begin{array}{c}
0<s<1,0<u<1 / 2,(1-u)^{2}-s u>0, \\
(1-u)^{2}-(1-s) u>0,1-2 t+t u-s u=0
\end{array}\right\} .
\end{aligned}
$$


Fig. $4 \mathcal{R}^{*}$ (on the top) and its partition $\left(\mathcal{R}^{*}\left(\chi^{\varepsilon}\right)\right)_{\varepsilon \in\{-, 0,+\}}$
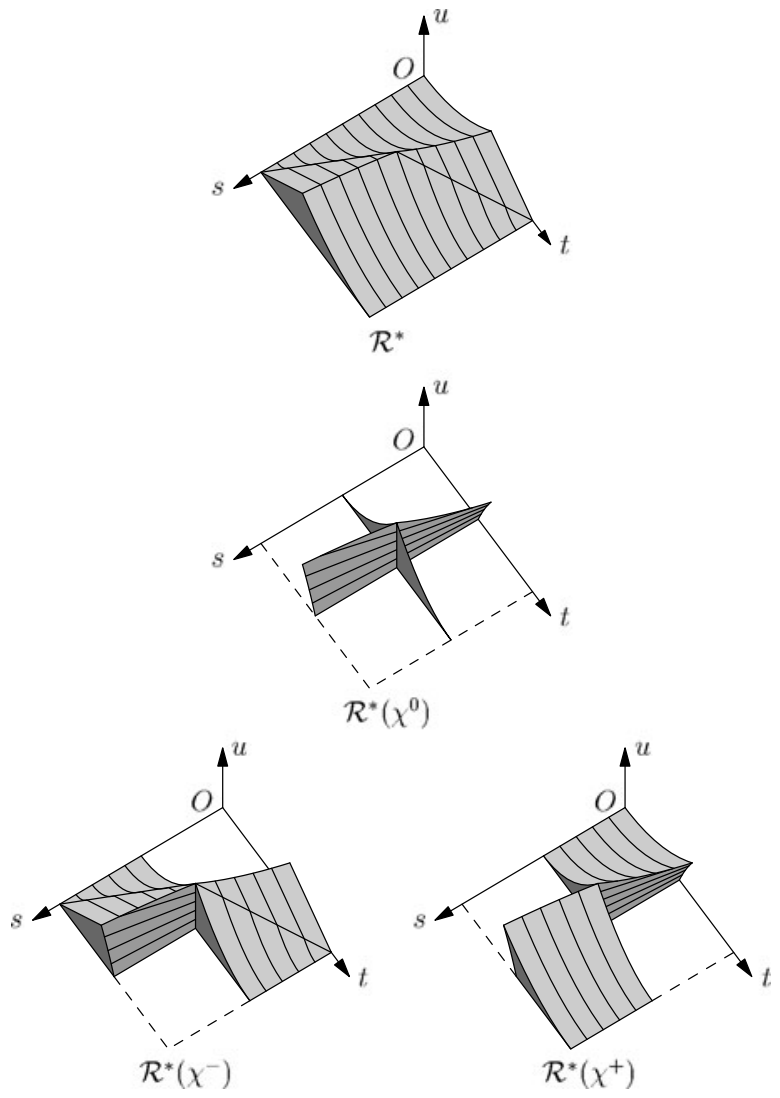

The intersection of the two irreducible components is the set

$$
\left\{(s, t, u) \in \mathbb{R}^{3} \mid s=t=\frac{1}{2}, 0<u<\frac{1}{2}\right\} \cong\left\{X\left(\frac{1}{2}, \frac{1}{2}, u\right) \mid 0<u<\frac{1}{2}\right\} .
$$

The proof is also similar to that of Proposition 2.2 and is omitted.

Figure 3 shows two realizations of $\mathcal{M}^{0}$. On the left, it shows the affine image of $X\left(\frac{1}{2}, \frac{3}{8}, \frac{1}{4}\right)$, on the irreducible component $1-2 s=0$. On the right, it shows the image of $X\left(\frac{3}{4}, \frac{11}{24}, \frac{2}{7}\right)$, on the other component $1-2 t+t u-s u=0$. These images can be deformed continuously to each other via $X\left(\frac{1}{2}, \frac{1}{2}, u\right)\left(0<u<\frac{1}{2}\right)$.

We set

$$
\mathcal{R}^{*}:=\left\{\begin{array}{l|c}
(s, t, u) \in \mathbb{R}^{3} \mid \begin{array}{c}
0<s<1,0<u<t<1-u \\
(1-t)^{2}-s u>0, t^{2}-(1-s) u>0
\end{array}
\end{array}\right\} .
$$

The set $\mathcal{R}^{*} \times(0, \infty)^{12}$ is rationally isomorphic to a realization space of a partial oriented matroid with the sign $\chi(9,12,13)$ non-fixed. The collection of semialgebraic sets $\left(\mathcal{R}^{*}\left(\chi^{\varepsilon}\right)\right)_{\varepsilon \in\{-, 0,+\}}$ is a partition of $\mathcal{R}^{*}$. Figure 4 illustrates this partition in 3-space. 
Acknowledgements I would like to thank Masahiko Yoshinaga for valuable discussions and comments. I also thank Yukiko Konishi for comments on the manuscript.

\section{References}

1. Björner, A., Las Vergnas, M., Sturmfels, B., White, N., Ziegler, G.: Oriented Matroids. Encyclopedia of Mathematics, vol. 46. Cambridge University Press, Cambridge (1993)

2. Günzel, H.: Universal partition theorem for oriented matroids. Discrete Comput. Geom. 15, 121-145 (1996)

3. Mnëv, N.E.: The universality theorems on the classification problem of configuration varieties and convex polytopes varieties. In: Viro, O.Ya. (ed.) Topology and Geometry-Rohlin Seminar. Lecture Notes in Mathematics, vol. 1346, pp. 527-544. Springer, Heidelberg (1988)

4. Richter-Gebert, J.: Mnëv's universality theorem revisited. Sémin. Lothar. Comb. 34, B34h (1995)

5. Richter-Gebert, J.: Two interesting oriented matroids. Doc. Math. 1, 137-148 (1996)

6. Ringel, G.: Teilungen der Ebene durch Geraden oder topologische Geraden. Math. Z. 64, 79-102 (1956)

7. Suvorov, P.Y.: Isotopic but not rigidly isotopic plane system of straight lines. In: Viro, O.Ya. (ed.) Topology and Geometry-Rohlin Seminar. Lecture Notes in Mathematics, vol. 1346, pp. 545-556. Springer, Heidelberg (1988) 